\title{
Resveratrol protects against protease inhibitor-induced ROS production, reticulum stress and lipid raft perturbation
}

Olivia TOUZET ${ }^{1}$ and Alexandre PHILIPS ${ }^{1,2}$

${ }^{1}$ INSERM, ERI25, "Muscle and Pathologies" and Université Montpellier 1, UFR Médecine, EA4202, Montpellier, France

\section{${ }^{2}$ Corresponding Author}

Alexandre Philips, INSERM, ERI25, "Muscle and Pathologies", CHU A. de Villeneuve, 371 rue du Doyen G. Giraud, F-34295 Montpellier Cedex5, France. Tel: 334674152 36, Fax: 334674152 31, mail to: alexandre.philips@inserm.fr

\section{Financial support}

INSERM ERI 25, F-34295 Montpellier, France; Université Montpellier 1, Montpellier, France; Languedoc-Roussillon region, Montpellier, France; Agence Nationale de Recherche contre le Sida, Paris, France; Association Française contre les Myopathies, Paris, France

Short title: Resveratrol and PI-induced side effects

Total number of words: 5750 or 3354 (not including abstract, references and figure legends) 


\section{Abstract}

Objective: HIV protease inhibitors (PIs) have been successfully used in highly active anti-retroviral therapy of HIV-1 infection but their benefits are compromised by a number of clinically important adverse side effects. Several studies showed that PIs induce sarco/endoplasmic reticulum (SR/ER) stress and overproduction of reactive oxygen species (ROS) but the hierarchy of these events was never established in PItreated cells. Our objective was to determine whether ROS production and SR/ER stress were co-induced by PIs in human primary skeletal myotubes and whether antioxidant treatment with resveratrol could protect against PI-induced cellular damages. Design and Methods: We analyzed in cultures of primary human skeletal myotubes the effects of four PIs (atazanavir, lopinavir, ritonavir and saquinavir) on ROS production (by measuring the reduction of nitroblue tetrazolium and by fluorescence microscopy using CM-H2DCFDA), on SR/ER stress induction (by immunofluorescence and electron microscopy) and on the expression and localization at lipid rafts of Caveolin 3 and Flotillin 1, two major components of lipid rafts (by immunoblotting and biochemical experiments). Cells were co-incubated with resveratrol to assess its protective effects. Results: In myotubes, PIs increased ROS production, altered SR/ER morphology, increased expression of CHOP, a SR/ER stress marker, and decreased expression and localization at lipid rafts of Caveolin 3 and Flotillin 1. Importantly, these deleterious PI effects were inhibited by the antioxidant resveratrol. Conclusions: Our findings demonstrate a protective effect of resveratrol against PI-induced SR/ER stress in human myotubes.

Key Words: HIV, Protease Inhibitors, Antioxidant, membrane microdomain, muscle skeletal 


\section{Introduction}

HIV protease inhibitors (PIs) have been successfully used in highly active anti-retroviral therapy (HAART) of HIV-1 infection, the most effective treatment currently available. Incorporation of PIs in HAART has significantly reduced the morbidity and mortality and prolonged the lifespan of patients with HIV infection. PI benefits are unfortunately compromised by a number of clinically important adverse side effects [1]. Most patients on HAART develop a metabolic syndrome associated with partial lipodystrophy, hyperlipidemia, insulin resistance, premature atherosclerosis and myocardial infarction [2-6]. Moreover, these patients face a growing number of other age-related comorbidities, such as neurodegeneration, osteopenia and malignancies $[7,8]$. The cellular and molecular mechanisms underlying PI-associated metabolic abnormalities remain elusive but they seem to be related to overproduction of reactive oxygen species (ROS) [9], induction of endoplasmic reticulum (ER) stress and activation of the unfolded protein response (UPR) [10, 11].

In many cell models, such as pancreatic insulinoma cells, cultured vascular smooth muscle cells or fibroblasts, PIs induce intracellular ROS production that is prevented by co-treatment with the antioxidants thymoquinone [12] or N-acetyl cysteine [13]. In rat pancreatic insulinoma cells, a direct role of ROS in the inhibition of insulin secretion by nelfinavir is suggested because thymoquinone reverses the suppressive effect of nelfinavir [12]. However, the clear implication of oxidative stress in PI-induced metabolic syndrome remains to be established. PIs also affect the overall functions of ER compartments in human fibrosarcoma [14] and myeloma cells [14] and in mouse macrophages [15]. Specifically, they deplete ER calcium stores and induce ER stress 
leading to UPR activation and expression of the ER stress markers X-box Binding Protein 1 (XBP-1), activating transcription factor 4 (ATF-4) and C/EBP Homologous Protein (CHOP) [15]. These transcription factors operate as downstream components of UPR to protect ER against accumulation of unfolded/misfolded proteins (reviewed in [16]). UPR hinders accumulation of misfolded proteins in ER by facilitating the expression of ER chaperones, inhibiting protein entry into ER through mRNA translation arrest and by increasing the efficiency of the ER-associated machinery for protein degradation (ERAD) [16]. Sarcoplasmic reticulum (SR) and ER are crucial for proper folding and post-transcriptional modifications of many cell surface proteins, including lipid raft microdomains, which are important cell signaling platforms (reviewed in [17]. In osteoclasts, recruitment of c-src and TNF receptor-associated factor 6 (TARF-6) to lipid rafts is inhibited by ritonavir treatment through an unknown mechanism, which could be linked to ER stress [18].

Whether PIs inhibit protein localization at lipid rafts in other tissues and how PIs lead to accumulation of unfolded/misfolded proteins in ER lumen remain important questions to be elucidated as well as the precise implication of ROS production in these deleterious processes. To elucidate these questions, we treated cultures of primary human myoblasts after they fused to form mature differentiated myotubes with the PIs atazanavir, lopinavir, ritonavir and saquinavir. Such treatment increased ROS production, modified SR/ER morphology and induced SR/ER stress in myotubes. Moreover, these PIs decreased the expression and localization at lipid rafts of Caveolin 3 and Flotillin 1, two major components of lipid raft microdomains [19-22]. To evaluate the role of ROS production in these deleterious effects, we used the antioxidant resveratrol, a stilbene found naturally in various plants with the highest concentration in 
grape skin and peanuts $[23,24]$. Resveratrol supplementation protects against ischemia/reperfusion injury [25-27] and attenuates many age-related illnesses [25, 2830], such cardiovascular disease, various types of cancers, neurodegeneration and type II diabetes, making of it a particularly attractive molecule to attenuate PI-induced cellular damage. Following co-treatment with resveratrol, PI deleterious effects on myotubes were inhibited, suggesting that SR/ER stress and lipid raft perturbation depend on ROS overproduction. 


\section{Methods}

Reagents

Saquinavir, ritonavir, lopinavir and atazanavir were purchased from Sequoia Research Products (Pangbourne, United Kingdom). Thapsigargin and resveratrol were purchased from Sigma Aldrich (St.Quentin Fallavier, France), Nitro-Blue-Tetrazolium (NBT) from Merk (Darmstadt, Germany) and CM-H2DCFDA derivatives (5 (and 6) chloromethyl-2',7'dichlorodihydrofluorescein diacetate, acetyl ester) from Invitrogen (Cergy Pontoise, France).

Anti- $\alpha$ Tubulin monoclonal antibody (mAb) was from Sigma Aldrich (St Quentin Fallavier, France); anti-CHOP mAb was from Cell Signaling Technology (Ozyme, Montigny, France); anti-Bip/GRP78, -Flotillin-1, -Caveolin-3 were from BD Transduction Laboratories (Franklin Lakes, New Jersey, USA). The Alexa Fluor secondary antibodies were from Invitrogen (Cergy Pontoise, France).

Primary cell cultures

Primary human myoblasts were isolated as described previously [31] from quadriceps muscle biopsies obtained from the "AFM-BTR Banque de Tissus pour la Recherche" (Hôpital de la Pitié-Salpêtrière, 75013 Paris). 30000 myoblasts/ml were grown in DMEM (Cambrex, Lonza, Verviers, Belgium) supplemented with 10\% fetal calf serum (FCS) (Perbio Science, Brebieres, France), 1\% UltroserG (Biosepra CI Pherben, Cergy St. Christophe, France) and Penicillin/Streptomycin (Cambrex, Lonza, Verviers, Bergium) for five days. At day 5, myoblasts started to fuse and cultures were switched to differentiation medium (DM) composed of DMEM supplemented with $10 \%$ FCS and Penicillin/Streptomycin for 4 days to obtain terminally differentiated myotubes that 
were then used for all the experiments. PIs were dissolved in dimethyl sulfoxide (DMSO) with $0.05 \%$ BSA and the final concentration of DMSO in DM was $<0.1 \%$; resveratrol was dissolved in ethanol and the final concentration of ethanol in DM was $<0.1 \%$. In control, cells were incubated with DMSO and/or ethanol. Cell viability was assessed by measuring cellular ATP levels using the ATPLite luminescence assay (PerkinElmer, Courtaboeuf, France).

ROS production

ROS production was detected by measuring NBT reduction. Cells were treated with PIs and 25 and $50 \mu \mathrm{M}$ resveratrol, or not, for 18 hours before incubation with $0.2 \%$ NBT in DMEM for 90 minutes. Then cell extracts were prepared as described previously [32] and the amount of dark-blue reduced NBT in extracts in $50 \%$ acetic acid was assessed at $560 \mathrm{~nm}$ and the optical density values were normalized to protein concentration measured with the bicinchoninic acid assay. We also used CM-H2DCFDA as cellpermeant indicator for ROS. Myotubes were cultured in 6-well plates, treated with PIs and $25 \mu \mathrm{M}$ resveratrol, or not, for 8 hours, before being incubated with $5 \mu \mathrm{M}$ CMH2DCFDA and DAPI in PBS at $37^{\circ} \mathrm{C}$ in the dark for $60 \mathrm{~min}$. Cells were then washed with PBS three times, fixed in $4 \%$ paraformaldehyde and stored at $4{ }^{\circ} \mathrm{C}$ for up to $16 \mathrm{~h}$ before image acquisition. Images were taken of at least 10 different regions with a Zeiss fluorescent microscope (AxioCamMRm equipped with a charged-coupled device camera Zeiss AxioCam MRm) and fluorescence quantification was performed using Image J (available at: rsb.nih.gov/ij/). All experiments were performed in triplicate.

Immunoblotting, immunofluorescence, electron microscopy 
Cell extracts prepared as described previously [31] were subjected to SDS-PAGE, blotted onto nitrocellulose membranes and probed with the indicated antibodies. Bound antibodies were detected with a chemiluminescence detection kit (PerkinElmer, Courtaboeuf, France). Analysis and quantification were performed with Kodak 1D3.6 software. For immunofluorescence, cells were fixed in 4\% paraformaldehyde in PBS and permeabilized with $0.1 \%$ Triton X-100 in PBS. Cells were incubated with primary antibodies for $2 \mathrm{~h}$, followed by Alexa Fluor secondary antibodies and DAPI staining. Cell imaging was performed at the "Centre Regional d'Imageries Cellulaire de Montpellier" with a Leica DM6000 microscope (Wetlar, Germany) using a 40x/1.30 EC Plan-Neofluar grade oil objective. Images were captured as 16 TIFF files with a Micro Max 1300 CCD camera (RS-Princeton Instruments Inc.) driven by MetaMorph (version 7; Universal Imaging Corp.). Images were processed using ImageJ.

For ultrastructural evaluation, primary human myotubes were immersed in a solution of $3.5 \%$ glutaraldehyde in Sorensen's buffer $(0.1 \mathrm{M}, \mathrm{pH} 7.4)$ at $4^{\circ} \mathrm{C}$ overnight. They were then rinsed in Sorensen's buffer and post-fixed in $1 \%$ osmic acid and $0.8 \%$ potassium ferrocyanide in the dark and at room temperature for $2 \mathrm{~h}$. After two rinses in Sorensen's buffer, cells were dehydrated in a graded series of ethanol solutions (30-100\%) and then embedded in EmBed 812. Thin sections ( $85 \mathrm{~nm}$; Leica-Reichert Ultracut E) were cut at different levels of each block, counterstained with uranyl acetate and observed using a Hitachi 7100 transmission electron microscope at the Centre de Ressources en Imagerie Cellulaire de Montpellier (France).

Isolation of detergent-resistant membrane (DRM) fractions 
Ten $10 \mathrm{~cm}$ dishes of myotubes were harvested, then centrifuged and cell pellets suspended in ice-cool lysis buffer containing 1\% Triton X-100. After measurement of the total protein concentration with the bicinchoninic acid assay, cell extracts were fractionated on a $4 \mathrm{ml}$ sucrose gradient [33]. Ten fractions were collected from the top. Thirty microliters of each fraction was analyzed by immunoblotting for Caveolin 3 , Flotillin 1, Tubulin $\alpha$ and Actin expression.

Statistical analysis

All quantitative data are presented as means \pm s.e.m. Statistical analysis to determine significance was performed using the Student's $t$-test. Values of $\mathrm{P} \leq 0.05$ were considered significant. 


\section{Results}

\section{Resveratrol protects against PI-induced production of ROS in human myotubes.}

As ROS induce cellular damage that could mediate PI-mediated metabolic syndrome, ROS production was investigated in cultures of primary human myoblasts that fused in vitro to form mature differentiated myotubes. Myotubes were treated with four PIs (20 $\mu \mathrm{M}$ atazanavir, $20 \mu \mathrm{M}$ lopinavir, $40 \mu \mathrm{M}$ ritonavir and $20 \mu \mathrm{M}$ saquinavir) for 18 hours and ROS production was evaluated indirectly by measuring NBT reduction. ROS production was significantly increased in PI-treated cells in comparison to untreated control cells (Fig. 1A). Moreover, ROS production in PI-treated myotubes was inhibited by co-incubation with resveratrol in a dose-dependent manner (Fig.1A), using noncytotoxic resveratrol concentrations (data not shown). These results were confirmed using the fluorescent probe CM-H2DCFDA. Dichloro-fluorescein oxidation increased twofold in myotubes treated with PIs for 8 hours compared to control cells (Fig. 1B) and again this effect was significantly inhibited by anti-oxidant treatment with $25 \mu \mathrm{M}$ resveratrol (Fig. 1B), suggesting that resveratrol could protect against ROS-mediated iatrogenic effects of PIs.

\section{Resveratrol protects against PI-induced sarco/endoplasmic reticulum stress in} human myotubes.

Accumulation of Prelamin A following PI-dependent inhibition of the ER metalloproteinase ZMPSTE24 was proposed to mediate PI-associated iatrogenic effects in fibroblasts. Thus, maturation of Lamin A was assessed in proliferating myoblasts and differentiated myotubes. Treatment with $20 \mu \mathrm{M}$ lopinavir, $40 \mu \mathrm{M}$ ritonavir and $20 \mu \mathrm{M}$ 
saquinavir for 18 hours induced Prelamin A accumulation in myoblasts (Fig. 2A), like in fibroblasts, but not in myotubes (Fig. 2A), suggesting that PI-induced iatrogenic effects are independent of Prelamin A accumulation in human myotubes. Next, we analyzed PI effects on SR/ER integrity because ROS can alter cellular redox reactions that interfere with protein disulphide bonding and result in protein misfolding leading to SR stress and UPR activation (reviewed in [16]). Following incubation with atazanavir, lopinavir, ritonavir and saquinavir (same concentrations as before) and $25 \mu \mathrm{M}$ resveratrol, or not, SR/ER distribution was monitored by immunodetection of BiP, a major ER chaperone protein and by high-resolution electron microscopy. In untreated myotubes, BiP was detected in the entire cell confirming the broad distribution of SR/ER (Fig. 2B) and electron microscopy showed the typical SR/ER morphology as flattened sacs (Fig. 2C). In myotubes treated with PIs for 18 hours or with $100 \mathrm{nM}$ of thapsigargin (TG), an irreversible inhibitor of the ER Ca2+-ATPase that induces SR/ER stress, for 4 hours (Fig. 2B and 2C) the broad expression of BiP was lost. Moreover, $\mathrm{SR} / \mathrm{ER}$ vacuolization, observed in the immunofluorescence images (arrows in Fig. 2B: addition of arrows will facilitate reading), was confirmed by electron microscopy (arrows in Fig. 2C). On the other hand, co-incubation with resveratrol protected against PI-induced SR/ER morphological alterations (Fig. 2B and 2C), suggesting that they, at least in part, are due to ROS overproduction.

We next tested whether PIs could induce SR/ER stress and activate UPR in human myotubes. To this aim, myotubes were incubated with PIs for 18 hours and with TG for 4 hours. Expression of CHOP, which is a key downstream transcription factor of UPR activated during SR/ER stress (Fig. 3A), strongly increased in TG- and in PI-treated myotubes. Co-incubation with $25 \mu \mathrm{M}$ resveratrol (Fig. 3B), however, inhibited CHOP 
upregulation in PI-treated myotubes (from 80-90\% of CHOP-positive nuclei to 9-13\%). Thus, induction of SR/ER stress by PIs is hindered by resveratrol.

\section{Resveratrol protects against PI-dependent inhibition of Caveolin 3 and Flotillin 1 expression and localization to lipid rafts.}

In an initial step to reestablish homeostasis and normal SR/ER function, UPR leads to the export of unfolded or misfolded SR/ER proteins to the cytosol for degradation. As $\mathrm{SR} / \mathrm{ER}$ is crucial for proper folding and post-transcriptional modifications of many cell surface proteins, including lipid raft microdomains, which are important cell signaling platforms, we assessed the effect of PIs on the expression of Caveolin 3 and Flotillin 1, two lipid raft components. Treatment with different concentrations (from 5 to $20 \mu \mathrm{M}$ ) of PIs for 18 and 24 hours reduced expression of Caveolin 3 and Flotillin 1 in myotubes (Fig. 4A for atazanavir and data not shown for the other PIs). Moreover, this decrease was dose- and time-dependent in human myotubes and was obtained at therapeutic concentrations (Fig. 4B). As for the previously described PI effects, co-treatment with different concentrations (from 5 to $25 \mu \mathrm{M}$ ) of resveratrol for 18 hours inhibited reduction of Caveolin 3 expression in myotubes in a dose-dependent manner (Fig. 4C and 4D).

To determine whether PIs also affected Caveolin 3 and Flotillin 1 localization at lipid raft microdomains, Triton X-100 lysates of human myotubes, which had been treated with $20 \mu \mathrm{M}$ of atazanavir and $25 \mu \mathrm{M}$ of resveratrol (or not), were fractionated on sucrose density gradients. Buoyant density and resistance to solubilization by Triton X100 at $4^{\circ} \mathrm{C}$ are widely used to isolate raft membranes. In these preparations, Caveolin 3 and Flotillin 1 retain their association with lipids and they are frequently used as lipid 
raft markers. Indeed they strongly accumulated in fractions 3-5 (DMR fractions) of control cells (DMSO alone) (Fig. 5A), but less in those of atazanavir-treated myotubes (Fig. 5A). Again, resveratrol reduced this PI effect (Fig. 5A). Western blot analysis of pooled fractions 3-5 (LR: lipid raft fractions) and pooled fractions 8-10 (NR: no raft fractions) (Fig. 5B) confirmed that localization of Caveolin 3 and Flotillin 1 at lipid rafts was impaired in myotubes treated with atazanavir, lopinavir, ritonavir and saquinavir and that this PI effect was hindered by resveratrol (Fig. 5B). These results indicate that PIs decrease the expression and membrane localization of two important components of lipid raft microdomains. Moreover, resveratrol protects against PIinduced loss of these proteins in lipid raft microdomains, suggesting that this antioxidant could attenuate PI-mediated iatrogenic effects. 


\section{Discussion}

In this work we show that PI treatment induces ROS production, SR/ER damage, SR stress and reduces Caveolin 3 and Flotillin 1 localization at lipid rafts. Co-treatment with the anti-oxidant resveratrol protects against these effects.

Our results suggest that, at least in myotubes, PI side effects are not due to defects in Prelamin A conversion. A role for Lamin A/C metabolism in PI-associated metabolic/lipodystrophy syndrome and premature ageing was proposed because PIs block the zinc metalloproteinase ZMPSTE24, an ER protein localized at the cytosolic surface of ER membranes, thus causing accumulation of farnesylated Prelamin A [3436]. Retention of the farnesylated residue confers toxic properties to the partially processed Prelamin A [37-39] and genetic defects in the conversion of Prelamin A to mature Lamin A (e.g., ZMPSTE24 deficiency) cause lipodystrophy and premature ageing syndromes, including the Hutchinson-Gilford progeria syndrome [40-42]. However, our results suggest that, at least in myotubes, PI side effects are due to another mechanism.

Our work indicates that ROS overproduction, SR/ER stress and lipid raft perturbation were co-induced by PI treatment in human primary myotubes. The deleterious effects of HIV PIs had been previously associated with ROS overproduction and ER stress but the hierarchy between these two events was not known. In human aortic endothelial cells, PIs alone and as part of HAART regimens increase ROS production [9]. Nelfinavir induces oxidative stress and can also lead to adipocyte necrosis [43] and premature senescence in human fibroblasts [34]. The clear implication of oxidative stress in PIinduced cellular damages is demonstrated by co-treatment with antioxidants. In 3T3-L1 adipocytes, the superoxide dismutase-mimetic antioxidant MnTBAP protects against 
nelfinavir-induced insulin resistance and cleavage of poly ADP ribose polymerase, a marker of apoptosis [13]. In rat pancreatic insulinoma cells, nelfinavir-dependent inhibition of glucose-stimulated insulin secretion is prevented by thymoquinone [12], a component of black seed oil with potent antioxidant properties. Similarly, we show that atazanavir, lopinavir, ritonavir and saquinavir induce ROS overproduction and SR/ER stress (SR/ER swelling, CHOP expression and loss of Caveolin 3 and Flotillin 1 expression) in differentiated myotubes. Induction of ER stress by PIs was already shown in cancer cell lines $[10,11,14]$ and in macrophages [15], but the potential implication of ROS production on the induction of ER stress was not investigated in these studies. Our results clearly suggest that PI-induced SR/ER stress and UPR are mediated by ROS overproduction because the antioxidant resveratrol protects against SR/ER swelling, CHOP upregulation and loss of Caveolin 3 and Flotillin 1 expression. PI-induced oxidative stress could induce SR/ER stress through different mechanisms. Nitric oxide (NO) and other ROS may modify oxidizable residues (cysteine, tyrosine) of SR-associated calcium channels, such as RyR (S-nitrosylation), and SR/ER Ca ${ }^{2+}$ ATPases (by tyrosine nitration) leading to SR/ER calcium depletion, which is a cause of protein misfolding [44-46] and consequently of SR/ER stress. Such mechanism could explain how ritonavir, atazanavir and indinavir decreased ER calcium content in a dosedependent manner in mouse macrophages [15]. In addition, production of NO, a mediator of protein nitrosylation, and of other ROS that alter cellular redox-dependent reactions interferes with protein disulphide bonding and results in misfolding of proteins which accumulate in ER lumen [47]. Interestingly, our results show that PIs inhibit localization of Caveolin 3 and Flotillin 1 at lipid raft microdomains. These two proteins are palmitoylated on cysteine residues in association with the ER and this post- 
translational modification is needed for their localization at lipid rafts microdomains (reviewed in $[19,48]$. Caveolin 3 and Flotillin 1 are two major components of lipid rafts and are implicated in insulin signaling to trigger glucose transporter redistribution in adipocytes and myotubes [49]. Thus, inhibition of their recruitment to lipid rafts by PIs could play an important role in PI-induced insulin resistance. The PI effect on lipid rafts is not restricted to myotubes and it has been described also in osteoclast precursors [18]. Ritonavir blocks osteoclastogenesis and osteoclast function by disrupting the recruitment of TARF6/c-Src complex to lipid rafts by an unidentified mechanism [18]. In myotubes, resveratrol protects against degradation and mislocalization of Caveolin 3 and Flotillin 1 induced by PIs, suggesting that these cellular damages are mediated by SR/ER stress and oxidative stress. Resveratrol holds a number of reported health benefits (cardioprotective, antiaging, neuroprotective, antiviral and anticancer) [25-30] that make of it a particularly attractive molecule to attenuate PI-induced side effects.

Using the antioxidant resveratrol, we show that inhibition of PI-induced ROS overproduction protects against PI-induced SR/ER stress and mislocalization of lipid raft proteins in human myotubes. These results strongly suggest that resveratrol treatment may be used as a potential therapeutic agent to attenuate PI-induced side effects. 


\section{Acknowledgements}

The authors are grateful to Chantal Cazevieille and Cécile Sanchez for their technical assistance and interpretation of data concerning ultrastructural evaluation. We would like to thank the Tissue Bank for Research (MYOBANK-AFM) of the Association Française contre les Myopathies (AFM) for generously providing human muscle biopsies. This work was supported by grants to A.P. from AFM and the Agence Nationale de Recherche Contre le Sida. O.T. was supported by the ANRS.

A.P. was responsible for the overall conception and design of the study, drafting the article, and obtaining funding. O.T. collected and assembled data and performed data analysis. All coauthors were responsible for interpretation of the data, critical revision of the article, and all provided final approval of the article. 


\section{References}

1. Hui DY. Effects of HIV protease inhibitor therapy on lipid metabolism. Prog Lipid Res 2003,42:81-92.

2. Beregszaszi M, Jaquet D, Levine M, Ortega-Rodriguez E, Baltakse V, Polak M, Levy-Marchal C. Severe insulin resistance contrasting with mild anthropometric changes in the adipose tissue of HIV-infected children with lipohypertrophy. Int J Obes Relat Metab Disord 2003,27:25-30.

3. Bongiovanni M, Bini T, Chiesa E, Cicconi P, Adorni F, Monforte d'Arminio A. Lopinavir/ritonavir vs. indinavir/ritonavir in antiretroviral naive HIVinfected patients: immunovirological outcome and side effects. Antiviral Res 2004,62:53-56.

4. Carr A, Samaras K, Burton S, Law M, Freund J, Chisholm DJ, Cooper DA. A syndrome of peripheral lipodystrophy, hyperlipidaemia and insulin resistance in patients receiving HIV protease inhibitors. Aids 1998,12:F5158.

5. Holmberg SD, Moorman AC, Greenberg AE. Trends in rates of myocardial infarction among patients with HIV. $N$ Engl $J$ Med 2004,350:730-732; author reply $730-732$.

6. Koster JC, Remedi MS, Qiu H, Nichols CG, Hruz PW. HIV protease inhibitors acutely impair glucose-stimulated insulin release. Diabetes 2003,52:1695-1700.

7. Kohli R, Klein RS, Schoenbaum EE, Anastos K, Minkoff H, Sacks HS. Aging and HIV infection. $J$ Urban Health 2006,83:31-42. 
8. Senior K. Growing old with HIV. Lancet Infect Dis 2005,5:739.

9. Mondal D, Pradhan L, Ali M, Agrawal KC. HAART drugs induce oxidative stress in human endothelial cells and increase endothelial recruitment of mononuclear cells: exacerbation by inflammatory cytokines and amelioration by antioxidants. Cardiovasc Toxicol 2004,4:287-302.

10. Gills JJ, Lopiccolo J, Tsurutani J, Shoemaker RH, Best CJ, Abu-Asab MS, et al. Nelfinavir, A lead HIV protease inhibitor, is a broad-spectrum, anticancer agent that induces endoplasmic reticulum stress, autophagy, and apoptosis in vitro and in vivo. Clin Cancer Res 2007,13:5183-5194.

11. Pyrko P, Kardosh A, Wang W, Xiong W, Schonthal AH, Chen TC. HIV-1 protease inhibitors nelfinavir and atazanavir induce malignant glioma death by triggering endoplasmic reticulum stress. Cancer Res 2007,67:10920-10928.

12. Chandra S, Mondal D, Agrawal KC. HIV-1 protease inhibitor induced oxidative stress suppresses glucose stimulated insulin release: protection with thymoquinone. Exp Biol Med (Maywood) 2009,234:442-453.

13. Ben-Romano R, Rudich A, Etzion S, Potashnik R, Kagan E, Greenbaum U, Bashan N. Nelfinavir induces adipocyte insulin resistance through the induction of oxidative stress: differential protective effect of antioxidant agents. Antivir Ther 2006,11:1051-1060.

14. Kraus M, Malenke E, Gogel J, Muller H, Ruckrich T, Overkleeft H, et al. Ritonavir induces endoplasmic reticulum stress and sensitizes sarcoma cells toward bortezomib-induced apoptosis. Mol Cancer Ther 2008,7:1940-1948.

15. Zhou H, Pandak WM, Jr., Lyall V, Natarajan R, Hylemon PB. HIV protease 
inhibitors activate the unfolded protein response in macrophages: implication for atherosclerosis and cardiovascular disease. Mol Pharmacol 2005,68:690-700.

16. Kim I, $\mathrm{Xu} \mathrm{W}$, Reed JC. Cell death and endoplasmic reticulum stress: disease relevance and therapeutic opportunities. Nat Rev Drug Discov 2008,7:10131030.

17. Fantini J, Garmy N, Mahfoud R, Yahi N. Lipid rafts: structure, function and role in HIV, Alzheimer's and prion diseases. Expert Rev Mol Med 2002,4:122.

18. Wang MW, Wei S, Faccio R, Takeshita S, Tebas P, Powderly WG, et al. The HIV protease inhibitor ritonavir blocks osteoclastogenesis and function by impairing RANKL-induced signaling. J Clin Invest 2004,114:206-213.

19. Babuke $\mathrm{T}$, Tikkanen R. Dissecting the molecular function of reggie/flotillin proteins. Eur J Cell Biol 2007,86:525-532.

20. Bickel PE, Scherer PE, Schnitzer JE, Oh P, Lisanti MP, Lodish HF. Flotillin and epidermal surface antigen define a new family of caveolae-associated integral membrane proteins. $J$ Biol Chem 1997,272:13793-13802.

21. Morrow IC, Rea S, Martin S, Prior IA, Prohaska R, Hancock JF, et al. Flotillin1/reggie-2 traffics to surface raft domains via a novel golgi-independent pathway. Identification of a novel membrane targeting domain and a role for palmitoylation. $J$ Biol Chem 2002,277:48834-48841.

22. Williams TM, Lisanti MP. The caveolin proteins. Genome Biol 2004,5:214.

23. Dirks Naylor AJ. Cellular effects of resveratrol in skeletal muscle. Life Sci 2009,84:637-640. 
24. Penumathsa SV, Maulik N. Resveratrol: a promising agent in promoting cardioprotection against coronary heart disease. Can J Physiol Pharmacol 2009,87:275-286.

25. Das DK, Maulik N. Resveratrol in cardioprotection: a therapeutic promise of alternative medicine. Mol Interv 2006,6:36-47.

26. Imamura G, Bertelli AA, Bertelli A, Otani H, Maulik N, Das DK. Pharmacological preconditioning with resveratrol: an insight with iNOS knockout mice. Am J Physiol Heart Circ Physiol 2002,282:H1996-2003.

27. Ray PS, Maulik G, Cordis GA, Bertelli AA, Bertelli A, Das DK. The red wine antioxidant resveratrol protects isolated rat hearts from ischemia reperfusion injury. Free Radic Biol Med 1999,27:160-169.

28. Bureau G, Longpre F, Martinoli MG. Resveratrol and quercetin, two natural polyphenols, reduce apoptotic neuronal cell death induced by neuroinflammation. J Neurosci Res 2008,86:403-410.

29. Howitz KT, Bitterman KJ, Cohen HY, Lamming DW, Lavu S, Wood JG, et al. Small molecule activators of sirtuins extend Saccharomyces cerevisiae lifespan. Nature 2003,425:191-196.

30. Sun W, Wang W, Kim J, Keng P, Yang S, Zhang H, et al. Anti-cancer effect of resveratrol is associated with induction of apoptosis via a mitochondrial pathway alignment. $A d v$ Exp Med Biol 2008,614:179-186.

31. Coisy-Quivy M, Touzet M, Bourret A, Hipskind RA, Mercier J, Fort P, Philips A. TC10 controls human myofibril organization and is activated by the sarcomeric RhoGEF obscurin. J. Cell Sci. 2009,122.

32. Causeret M, Taulet N, Comunale F, Favard C, Gauthier-Rouviere C. N- 
cadherin association with lipid rafts regulates its dynamic assembly at cellcell junctions in C2C12 myoblasts. Mol Biol Cell 2005,16:2168-2180.

33. Iwabuchi K, Handa K, Hakomori S. Separation of "glycosphingolipid signaling domain" from caveolin-containing membrane fraction in mouse melanoma B16 cells and its role in cell adhesion coupled with signaling. $J$ Biol Chem 1998,273:33766-33773.

34. Caron M, Auclair M, Donadille B, Bereziat V, Guerci B, Laville M, et al. Human lipodystrophies linked to mutations in A-type lamins and to HIV protease inhibitor therapy are both associated with prelamin A accumulation, oxidative stress and premature cellular senescence. Cell Death Differ 2007,14:1759-1767.

35. Coffinier C, Hudon SE, Farber EA, Chang SY, Hrycyna CA, Young SG, Fong LG. HIV protease inhibitors block the zinc metalloproteinase ZMPSTE24 and lead to an accumulation of prelamin A in cells. Proc Natl Acad Sci U S A 2007,104:13432-13437.

36. Coffinier C, Hudon SE, Lee R, Farber EA, Nobumori C, Miner JH, et al. A potent HIV protease inhibitor, darunavir, does not inhibit ZMPSTE24 or lead to an accumulation of farnesyl-prelamin A in cells. $J$ Biol Chem 2008,283:9797-9804.

37. Fong LG, Ng JK, Lammerding J, Vickers TA, Meta M, Cote N, et al. Prelamin A and lamin A appear to be dispensable in the nuclear lamina. $J$ Clin Invest 2006,116:743-752.

38. Fong LG, Ng JK, Meta M, Cote N, Yang SH, Stewart CL, et al. Heterozygosity for Lmna deficiency eliminates the progeria-like phenotypes in Zmpste24- 
deficient mice. Proc Natl Acad Sci U S A 2004,101:18111-18116.

39. Young SG, Fong LG, Michaelis S. Prelamin A, Zmpste24, misshapen cell nuclei, and progeria--new evidence suggesting that protein farnesylation could be important for disease pathogenesis. $J$ Lipid Res 2005,46:2531-2558.

40. Agarwal AK, Fryns JP, Auchus RJ, Garg A. Zinc metalloproteinase, ZMPSTE24, is mutated in mandibuloacral dysplasia. Hum Mol Genet 2003,12:1995-2001.

41. Moulson CL, Go G, Gardner JM, van der Wal AC, Smitt JH, van Hagen JM, Miner JH. Homozygous and compound heterozygous mutations in ZMPSTE24 cause the laminopathy restrictive dermopathy. $J$ Invest Dermatol 2005,125:913-919.

42. Navarro CL, Cadinanos J, De Sandre-Giovannoli A, Bernard R, Courrier S, Boccaccio I, et al. Loss of ZMPSTE24 (FACE-1) causes autosomal recessive restrictive dermopathy and accumulation of Lamin A precursors. Hum Mol Genet 2005,14:1503-1513.

43. Vincent S, Tourniaire F, El Yazidi CM, Compe E, Manches O, Plannels R, Roche R. Nelfinavir induces necrosis of 3T3F44-2A adipocytes by oxidative stress. J Acquir Immune Defic Syndr 2004,37:1556-1562.

44. Viner RI, Ferrington DA, Williams TD, Bigelow DJ, Schoneich C. Protein modification during biological aging: selective tyrosine nitration of the SERCA2a isoform of the sarcoplasmic reticulum Ca2+-ATPase in skeletal muscle. Biochem J 1999,340 ( Pt 3):657-669.

45. Xu KY, Huso DL, Dawson TM, Bredt DS, Becker LC. Nitric oxide synthase in cardiac sarcoplasmic reticulum. Proc Natl Acad Sci U S A 1999,96:657-662. 
46. $\mathrm{Xu} \mathrm{L,} \mathrm{Eu} \mathrm{JP,} \mathrm{Meissner} \mathrm{G,} \mathrm{Stamler} \mathrm{JS.} \mathrm{Activation} \mathrm{of} \mathrm{the} \mathrm{cardiac} \mathrm{calcium}$ release channel (ryanodine receptor) by poly-S-nitrosylation. Science 1998,279:234-237.

47. Gotoh T, Mori M. Nitric oxide and endoplasmic reticulum stress. Arterioscler Thromb Vasc Biol 2006,26:1439-1446.

48. Fruhbeck G, Lopez M, Dieguez C. Role of caveolins in body weight and insulin resistance regulation. Trends Endocrinol Metab 2007,18:177-182.

49. Fecchi K, Volonte D, Hezel MP, Schmeck K, Galbiati F. Spatial and temporal regulation of GLUT4 translocation by flotillin-1 and caveolin-3 in skeletal muscle cells. FASEB J 2006,20:705-707. 


\section{Legends to figures}

Figure 1: Increased production of ROS in myotubes treated with PIs.

(A) Human myotubes were treated with vehicle alone $(\mathrm{Ctl})$ or with $20 \mu \mathrm{M}$ atazanavir (ATV), lopinavir (LPV), saquinavir (SQV) or $40 \mu \mathrm{M}$ ritonavir (RTV) and the indicated concentrations of resveratrol. After 18 hours, ROS production was measured in terms of NBT reduction and normalized to protein content. (B) Human myotubes were treated with vehicle alone $(\mathrm{Ctl})$ or with $20 \mu \mathrm{M}$ atazanavir, lopinavir, saquinavir or $40 \mu \mathrm{M}$ ritonavir and with $25 \mu \mathrm{M}$ resveratrol $(+)$ or not $(-)$ as indicated. After 8 hours, ROS production was assessed in terms of oxidation of CM-H2DCFDA derivatives (ROSinduced oxidation results in green labeling) as described in Methods. DMSO alone (final concentration $<0.1 \%$ ) or with ethanol (final concentration $<0.1 \%$ ) did not alter ROS production in control myotubes $(\mathrm{Ctl})$. Data are the means \pm s.e.m. of three independent experiments (\#, statistical significance relative to control; *, statistical significance relative to treatment, $\mathrm{P}<0.05)$.

\section{Figure 2: Resveratrol protects against PI-induced SR/ER vacuolization.}

(A) Human proliferating myoblasts or differentiated myotubes were treated with vehicle alone (Ctl) or with $20 \mu \mathrm{M}$ atazanavir (ATV), lopinavir (LPV), saquinavir (SQV) or $40 \mu \mathrm{M}$ ritonavir (RTV) as indicated. After 18 hours, cells were lysed and immunoblotted using the indicated antibodies. Analysis of $\beta$-Actin expression served as loading control. (B) Human myotubes were treated vehicle alone (Ctl) or with $20 \mu \mathrm{M}$ atazanavir

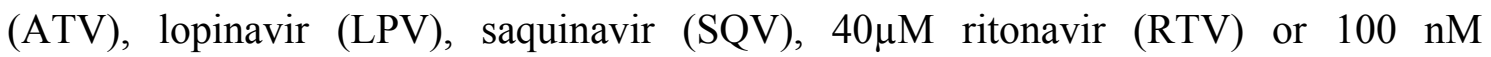


thapsigargin (TG), a known inducer of SR/ER stress, and with $25 \mu \mathrm{M}$ resveratrol $(+)$ or not (-) as indicated. After 18 hours, myotubes were fixed and incubated with anti-BiP antibodies, followed by fluorescently-labeled secondary antibodies. Scale bar: $5 \mu \mathrm{m}$. (C) Human myotubes were treated as described in (B) and after 18 hours, cells were fixed and analyzed by electron microscopy (X 10,000). Scale bar: $500 \mathrm{~nm}$.

\section{Figure 3: Resveratrol protects against PI-induced CHOP expression.}

(A) Human myotubes were treated vehicle alone (Ctl) or with $20 \mu \mathrm{M}$ atazanavir (ATV), lopinavir (LPV), saquinavir (SQV), $40 \mu \mathrm{M}$ ritonavir (RTV) or $100 \mathrm{nM}$ thapsigargin (TG), a known inducer of CHOP expression and SR/ER stress, and with $25 \mu \mathrm{M}$ resveratrol $(+)$ or not $(-)$ as indicated. After 18 hours, myotubes were fixed and incubated with anti-CHOP antibodies followed by fluorescently-labeled secondary antibodies (CHOP). DNA was stained with DAPI. (B) The percentage of CHOPpositive nuclei was determined in three independent experiments. Data are the means \pm s.e.m. of three independent experiments (\#, statistical significance relative to control $(\mathrm{Ctl})$ * $^{*}$, statistical significance relative to treatment, $\left.\mathrm{P}<0.05\right)$. Scale bar: $5 \mu \mathrm{m}$.

Figure 4: Resveratrol protects against PI-induced inhibition of Caveolin 3 expression.

(A) Human myotubes were treated with the indicated concentrations of atazanavir (ATV) for 18 or 24 hours. Next, myotubes were lysed and immunoblotted using the indicated antibodies. Analysis of $\beta$-Actin expression served as loading control. (B) 
Quantification of the expression level of the proteins obtained in (A) after 18 hours (black) and 24 hours (gray) incubation with different concentrations of ATV. Data are the means \pm s.e.m. of three independent experiments $(\mathrm{P}<0.05)$. (C) Human myotubes were treated vehicle alone (Ctl) or with $20 \mu \mathrm{M}$ atazanavir (ATV), lopinavir (LPV), saquinavir (SQV) or $40 \mu \mathrm{M}$ ritonavir (RTV) and with the indicated concentrations of resveratrol. After 18 hours, cells were lysed and immunoblotted using the indicated antibodies. Analysis of $\beta$-Actin expression served as loading control. (D) Quantification of Caveolin 3 expression obtained in (C). Data are the means \pm s.e.m. of three independent experiments (\#, statistical significance relative to control $(\mathrm{Ctl})$; *, statistical significance relative to treatment, $\mathrm{P}<0.05)$.

Figure 5: Resveratrol protects against PI-induced loss of Caveolin 3 and Flotillin 1 from lipid rafts.

(A) Human myotubes were treated vehicle alone (Ctl) or with $20 \mu \mathrm{M}$ atazanavir (ATV) and with $25 \mu \mathrm{M}$ resveratrol (+) or not (-) as indicated. After 18 hours, myotubes were lysed in $1 \%$ Triton X-100 and fractionated on a sucrose gradient as described in Methods. Thirty microliters of each fraction were resolved on SDS-PAGE gels and immunoblotted using the indicated antibodies. (B) Human myotubes were treated vehicle alone (Ctl) or with $20 \mu \mathrm{M}$ atazanavir (ATV), lopinavir (LPV), saquinavir (SQV) or $40 \mu \mathrm{M}$ ritonavir (RTV) and with $25 \mu \mathrm{M}$ resveratrol (+) or not (-) as indicated. After 18 hours, myotubes were lysed and fractionated as in (A). Fractions 3-5 (corresponding to lipid raft fractions (LR)) and fractions 8-10 (corresponding to non-lipid raft fractions 
(NR)) were pooled and Caveolin 3 and Flotillin 1 expression was assessed by immunoblotting. Analysis of $\beta$-Actin expression served as loading control. 

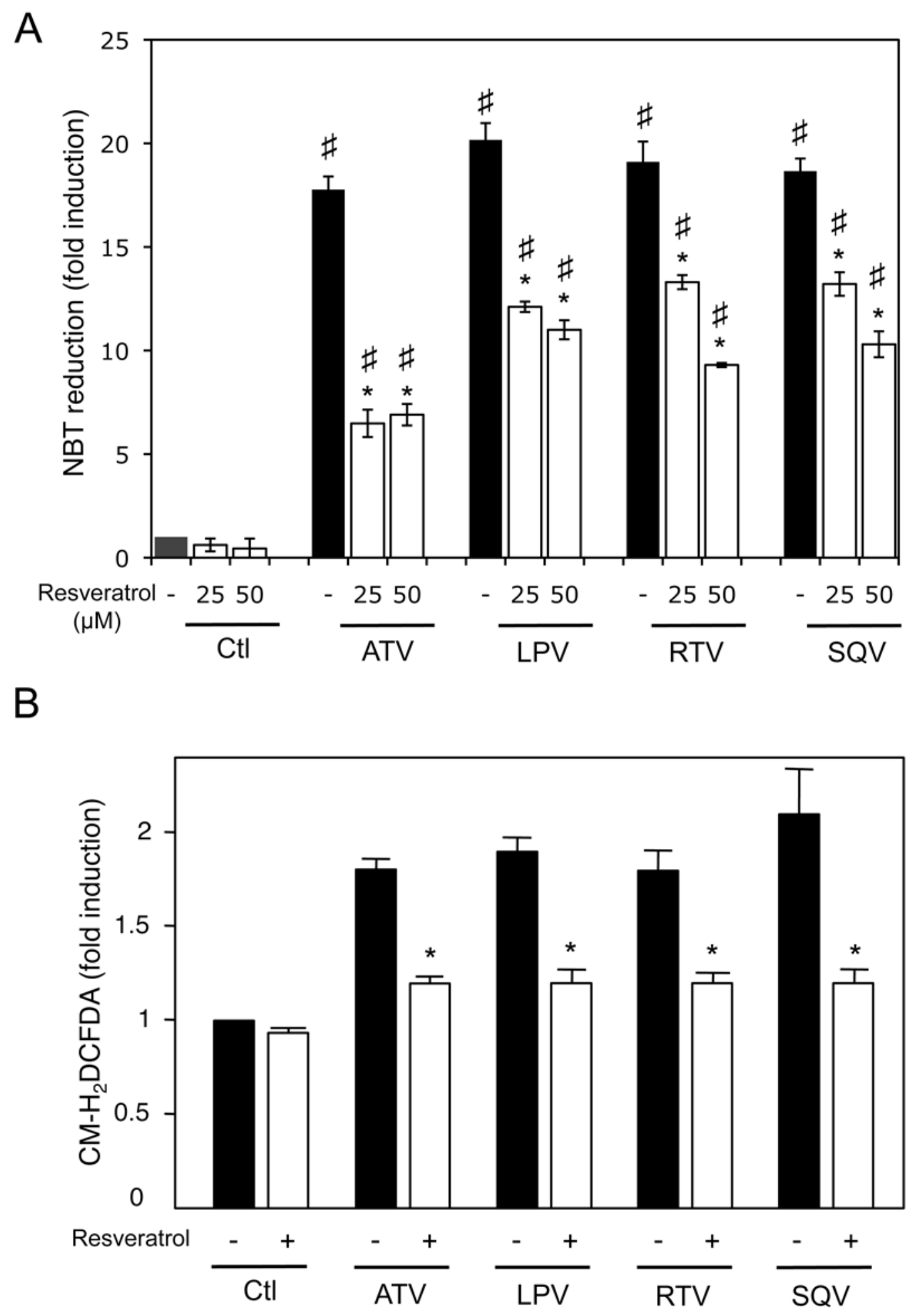
A

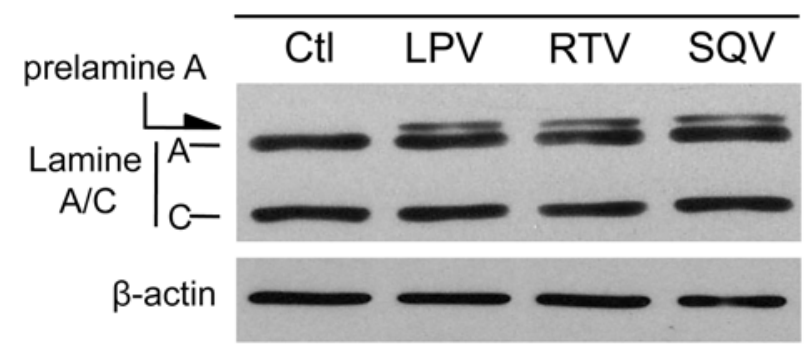

B

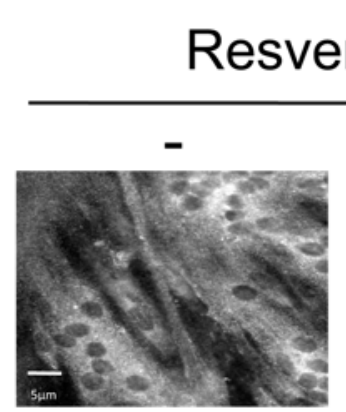

CtI
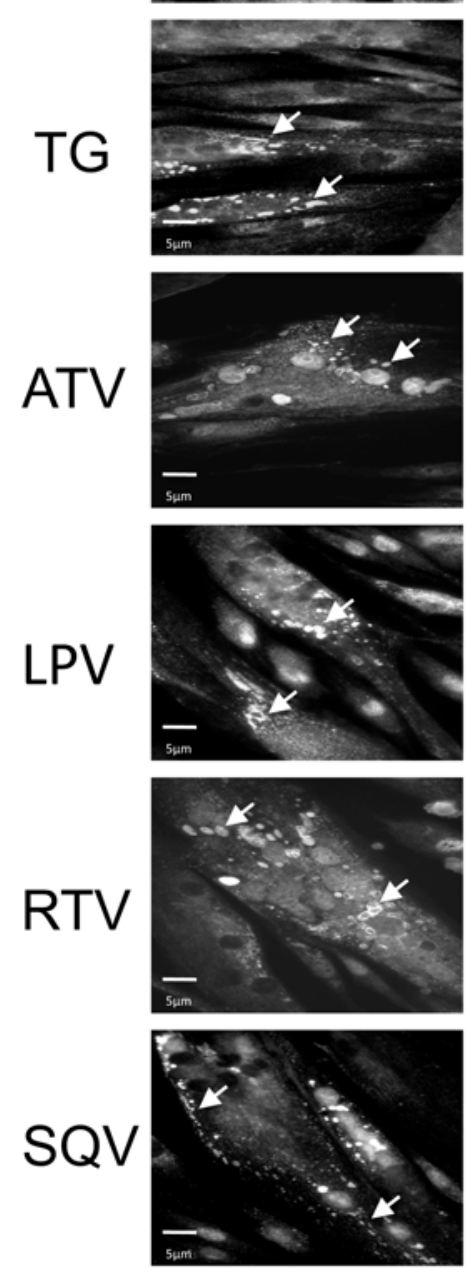

myoblasts
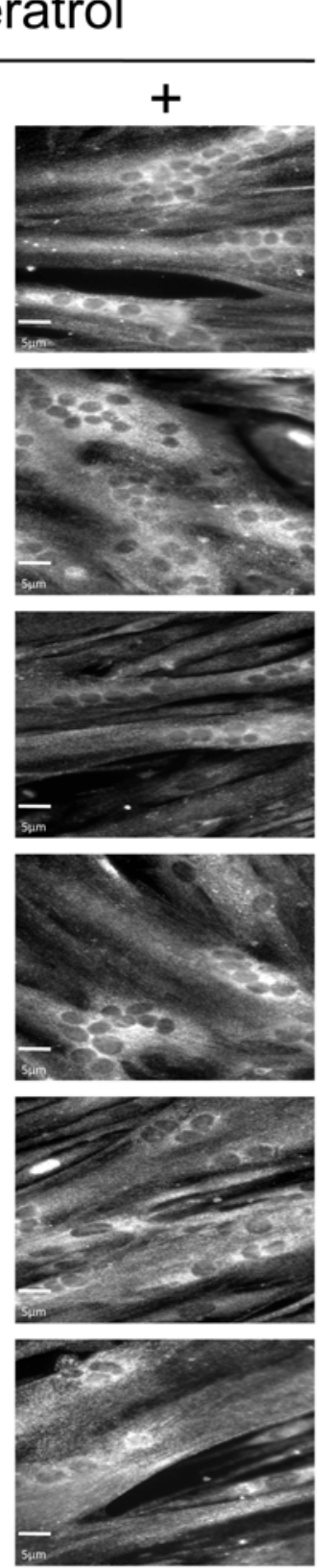

myotubes

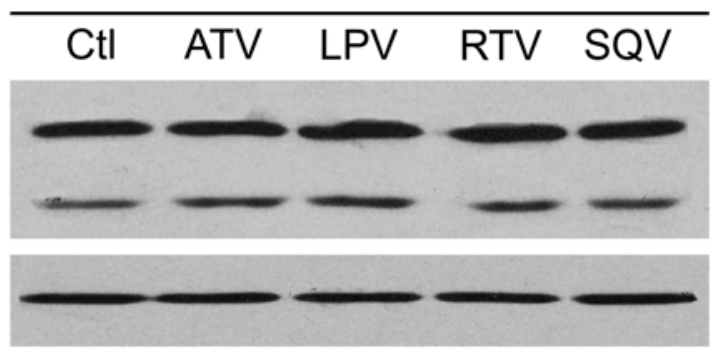

C

\section{Resveratrol}
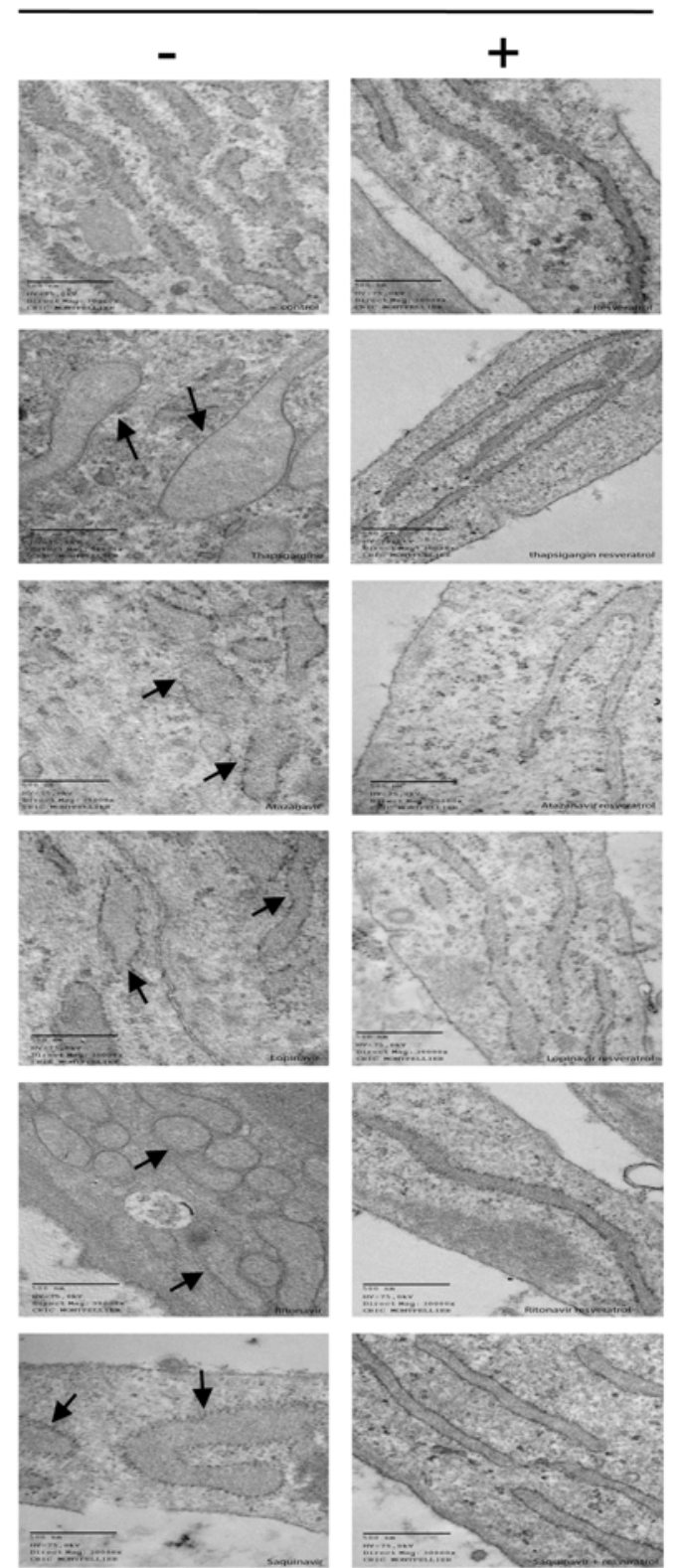

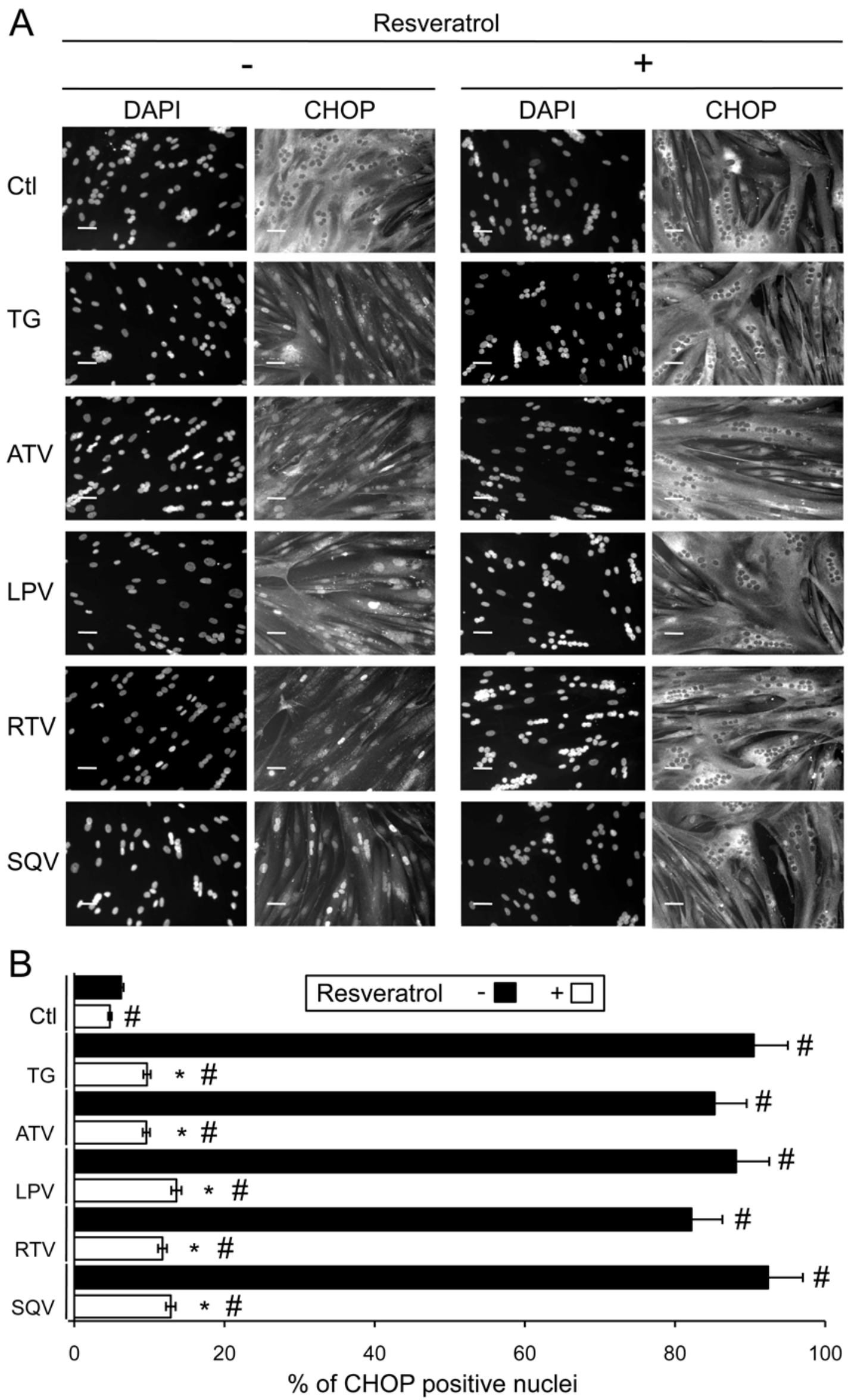


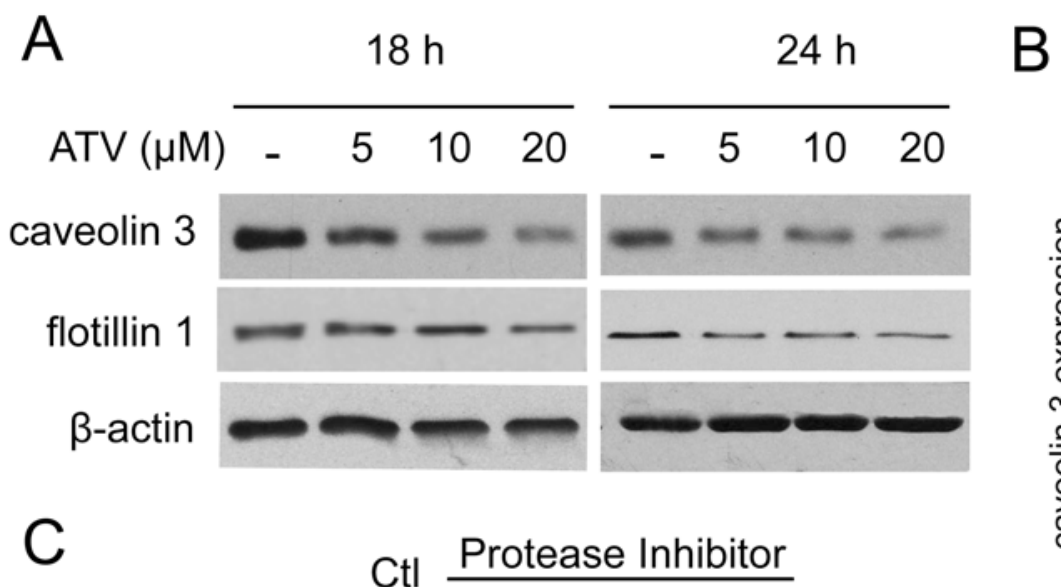

B $\square 18$ hours $\quad 24$ hours
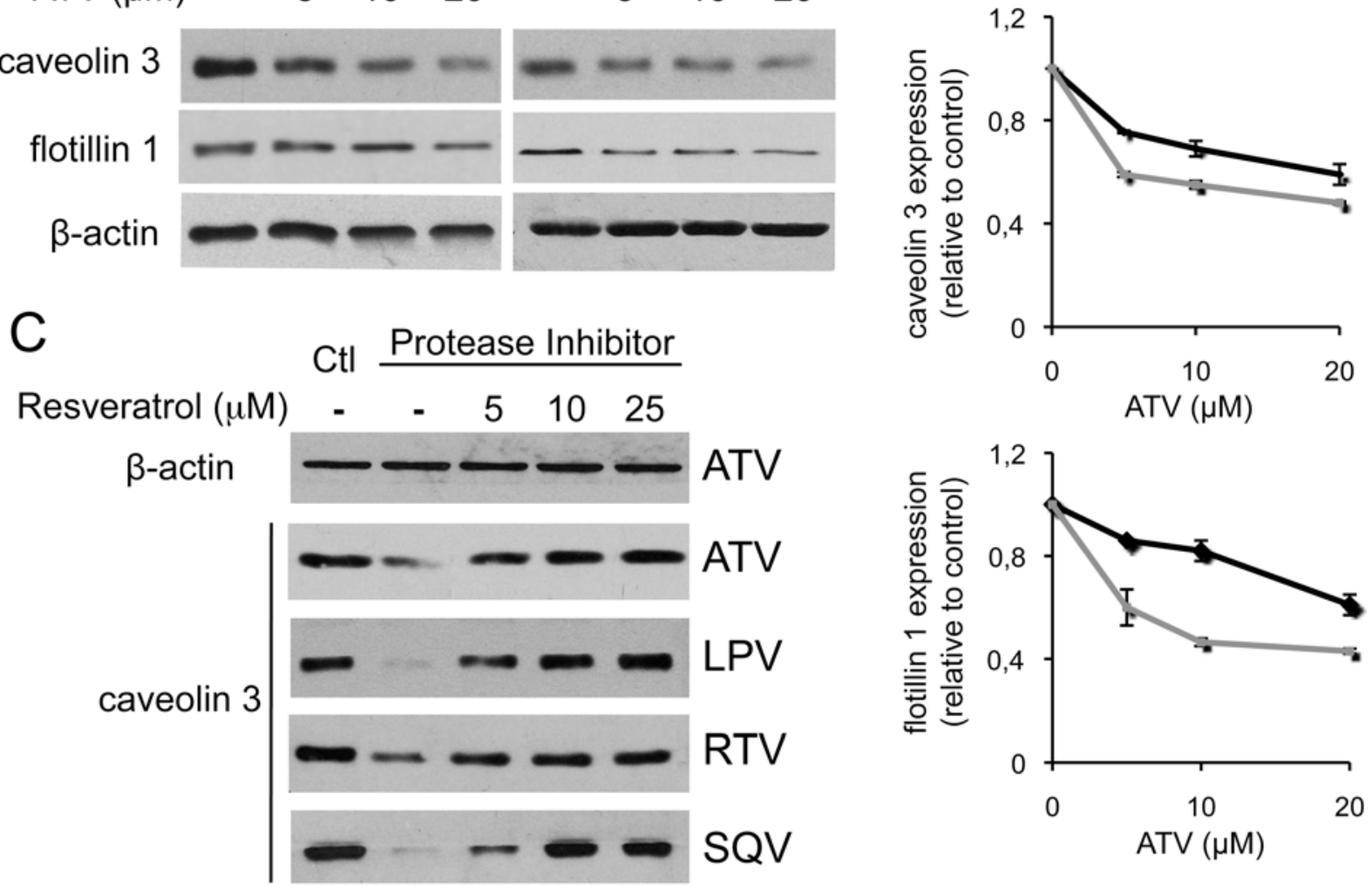

D

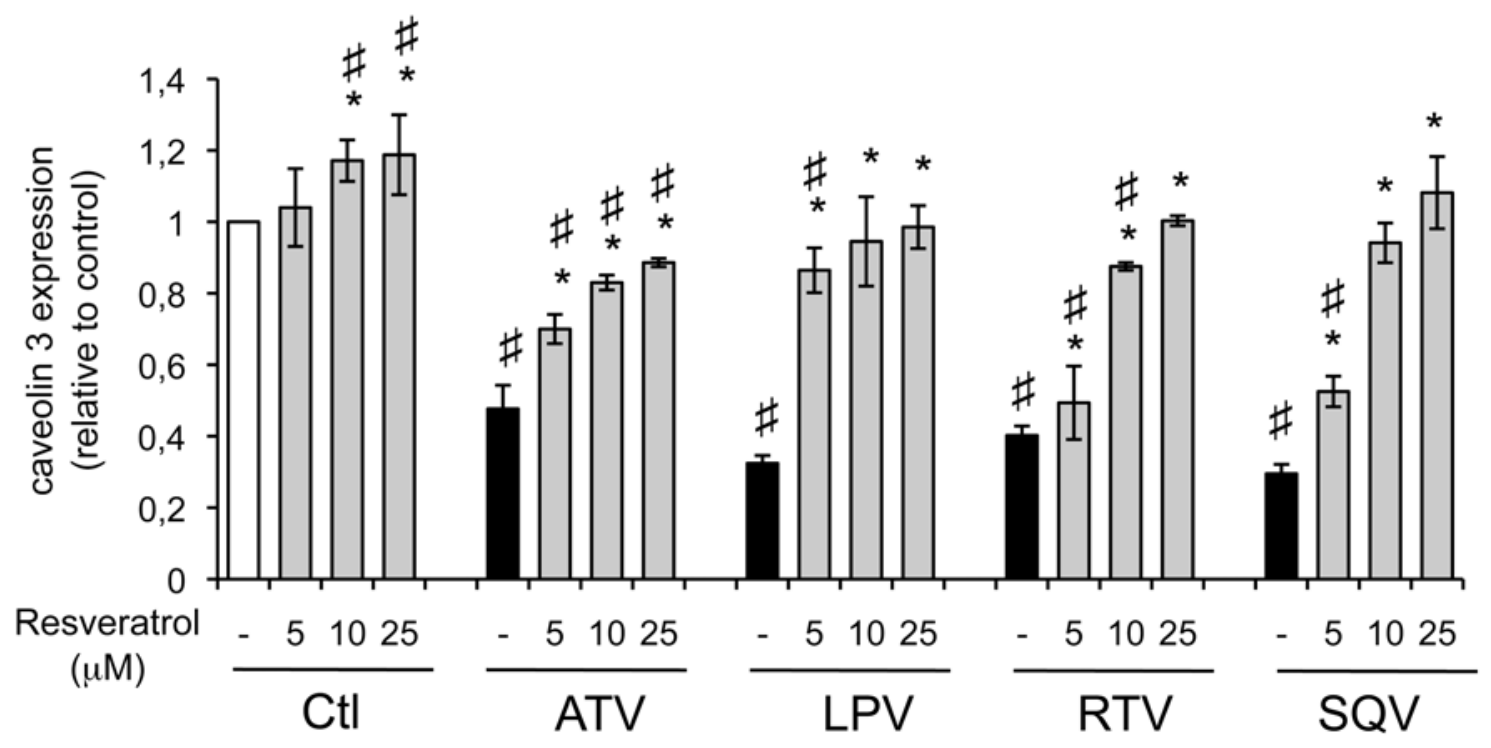


A Lipid Rafts
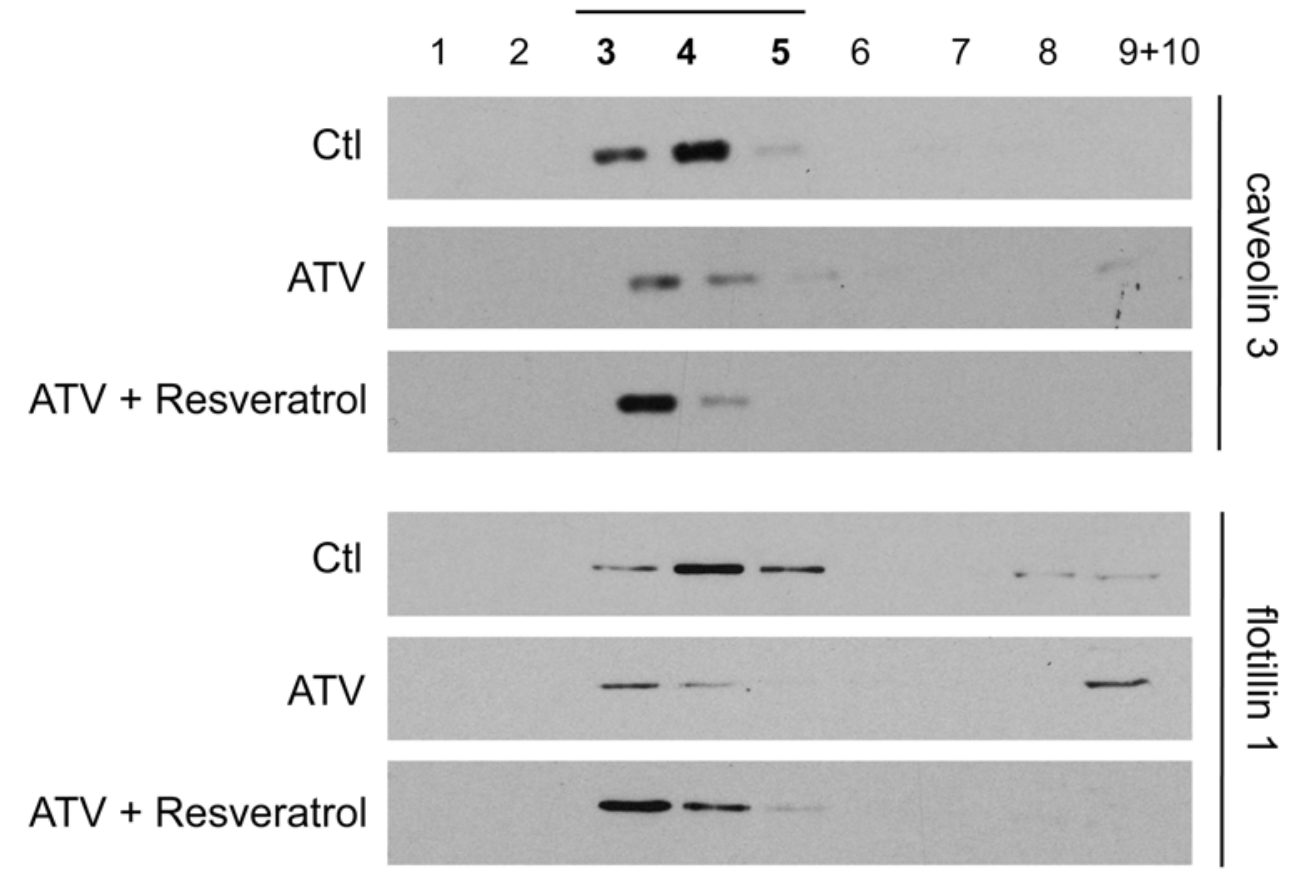

B

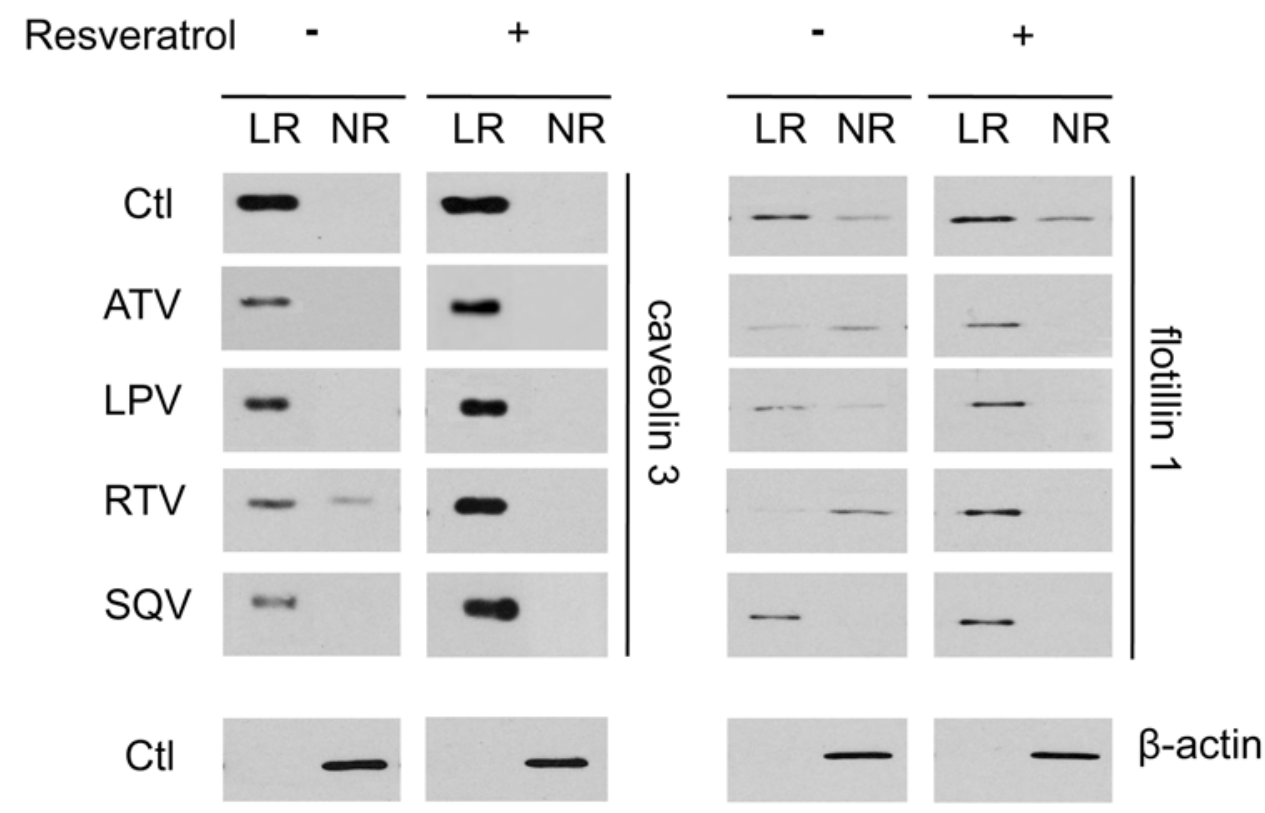

